\title{
Generating Valence Shifted Turkish Sentences
}

\author{
Seniz Demir \\ TUBITAK-BILGEM \\ Gebze, Kocaeli, TURKEY \\ seniz.demiratubitak.gov.tr
}

\begin{abstract}
Valence shifting is the task of rewriting a text towards more/less positively or negatively slanted versions. This paper presents a rule-based approach to producing Turkish sentences with varying sentiment. The approach utilizes semantic relations in the Turkish and English WordNets to determine word polarities and involves the use of lexical substitution and adverbial rules to alter the sentiment of a text in the intended direction. In a user study, the effectiveness of the generation approach is evaluated on real product reviews.
\end{abstract}

\section{Introduction}

Language can express a content in a number of different ways with varying emotion. Emotions might equip sentences with connotations and have a powerful effect on the disposition of the hearer in a subtle way. Moreover, emotions induced through words play an important role in written and verbal communication. Sentence valence specifies the degree of emotion present in a sentence and indicates how positive or negative the sentence is. The literature has shown that the sentiment characteristics of a sentence are correlated with the valence of words the sentence contains (Guerini et al., 2008).

Valence shifting is the task of altering a text towards more/less positively or negatively slanted versions while keeping much of its semantic meaning (Gardiner and Dras, 2012). This relatively new problem has many practical uses in language based applications such as persuasive systems which are designed to influence users' behaviors. Slanting of texts can be achieved in a number of ways, the most popular of which is the lexical substitution of semantically related words with varying valences (Whitehead and Cavedon, 2010). To our knowledge, this work is the first to examine the correlation between word polarities and sentence valences in Turkish and to address the problem of valence shifting in Turkish sentences. Our methodology for determining word polarities explores the semantic relations of words within and between the Turkish and English WordNets. To alter the sentiment carried by a sentence in the intended direction, our approach utilizes word polarities and a number of hand constructed rules based on the insights gained from user studies. Two strategies, namely lexical substitution and the use of intensifiers/downtoners, are used to slant Turkish texts. An evaluation study shows the effectiveness of our approach in generating valence shifted Turkish sentences.

\section{Word Polarity}

Word polarity (valence) stands for the semantic orientation of a word and is one of positive, negative or neutral. Previous research has shown that it is very common to retrieve word valences from existing polarity lexicons. To our best knowledge, there is only one available Turkish word polarity lexicon $\left(\operatorname{Tr} \_\mathbf{L}\right)$ which is built in a semi-automated manner by traversing a multilingual word relatedness graph with a random walk model (Özsert and Özgür, 2013). The lexicon consists of 1398 positive (e.g., "övgü\#n” (praise\#n)) and 1414 negative (e.g., "anormal\#a" (abnormal\#a)) word entries. Although all word entries are given along with their PoS (i.e., one of noun, verb, or adjective), the lexicon neither contains word senses nor the strength of polarities.

There are a number of available English polarity lexicons. The General Inquirer lexicon (GI_L) annotates word entries with syntactic, semantic, and pragmatic information including its sense and PoS (Stone et al., 1966). In the MPQA_Polarity lexicon (MPQA L), word entries are annotated with PoS, polarity, and the strength of polarity (i.e., strong or weak) but no sense information is 


\begin{tabular}{|l|l|l|l|l|}
\hline Polarity Agreement with Tr_L & GI_L & MPQA_L & SWN_L & En_L \\
\hline Positive polarity match & 646 & 468 & 423 & 950 \\
\hline Negative polarity match & 761 & 775 & 742 & 1339 \\
\hline No Turkish polarity \& Positive English polarity & 326 & 376 & 750 & 1177 \\
\hline No Turkish polarity \& Negative English polarity & 373 & 577 & 1019 & 1390 \\
\hline
\end{tabular}

Table 1: The agreement of word polarities.

given (MPQA, 2014). The SentiWordNet lexicon (SWN_L), along with PoS and sense information, annotates word entries with three sentiment scores from positivity, negativity, and objectivity perspectives (Esuli and Sebastiani, 2006) ${ }^{1}$.

Due to the limitations of the Turkish lexicon (e.g., no adverbs exist in the lexicon), we explored ways of expanding the scope of the lexicon by taking advantage of the semantic relations between words. As described in the rest of this section, we also examined how additional polarity information can be retrieved from English polarity lexicons and applied to Turkish.

\subsection{Bilingual WordNet Graph}

The Turkish WordNet is fully compatible with but not as comprehensive as some other WordNets such as EuroWordNet. We represent the Turkish WordNet as a directed graph where each vertex corresponds to a word tagged with the sense and PoS information (e.g., mekan\#1, $\mathrm{n}^{2}$ ). The vertices corresponding to the words that share a relation are connected with a directed edge and the edge is labeled with the kind of this relation (e.g., "synonym" or "antonym"). A monolingual graph consisting of 20343 vertices and 60164 edges is built from the Turkish WordNet. Following the same representation scheme, a monolingual graph is built from the English WordNet 2.0 which contains 177308 vertices and 786932 edges.

The Turkish and English monolingual graphs are integrated into a big bilingual graph with the use of the InterLingual Index (ILI) where words having the same meaning are connected. ILI facilitates the mapping of concepts and similar synsets between compatible WordNets. This integration enabled us to explore the agreement of word polarities between the Turkish polarity lexicon and each of the three English polarity lexicons. The first and the second rows in Table 1 show the number of cases where a Turkish-English word pair with the same ILI share a positive or a negative polarity re-

\footnotetext{
${ }^{1}$ Here, we classify a word as positive/negative if its positivity/negativity score is greater than or equal to 0.5 .

${ }^{2}$ The noun mekan (location) is of the first sense.
}

spectively. The third and the fourth rows represent the cases where a Turkish word does not have a polarity in the Turkish lexicon whereas its English correspondent has a positive or a negative polarity in the English lexicon respectively. For instance, the word "bitmek bilmemek\#a" does not have a polarity in $\operatorname{Tr} \_$whereas its English correspondent "endless\#a" has a negative polarity in MPQA_L.

We examined whether individual agreements between the Turkish lexicon and each English lexicon can be improved by merging all English lexicons into a single polarity lexicon (En $\mathbf{L})$. During this merge, words that have different polarities in individual lexicons are omitted and the words from MPQA $L$ are considered as of the first sense. The final En_L lexicon consists of 9044 positive and 13890 negative words with PoS and sense information. As shown in the fourth column of Table 1, this merge improves the agreement between the Turkish and English polarity lexicons.

\subsection{Detecting Word Polarity}

A two-step approach is developed for determining the polarities of Turkish words. Once given a sentence, this approach first identifies prior word polarities by utilizing the information contained in polarity lexicons and then applies a set of polarity alteration rules to the sentence for finalizing polarity assignments.

To determine the polarity of a word, the presence of the word and its synonyms is first explored in the Turkish polarity lexicon. If neither the word nor any of its synonyms exists in the $\operatorname{Tr} \mathrm{L}$ lexicon, English words that have the same ILI with the Turkish word are explored in the English polarity lexicon $E_{-} L^{3}$. If the word polarity is still not determined, the polarity of Turkish words that share the antonym relation with the word is explored in $\operatorname{Tr} \_$and the reverse of the retrieved word polarity (if any) is taken. If the use of antonym relation in $\operatorname{Tr} \mathrm{L}$ does not return a polarity, the antonym relation is explored in the En_L lexicon for the English correspondents of the Turkish word.

\footnotetext{
${ }^{3}$ This enables us to benefit from English polarities shown in the third and the fourth rows of Table 1 .
} 
We hand constructed a number of polarity alteration rules that are specific to Turkish. These rules, once applied to a sentence, might alter the lexicon-based prior polarity of words. For example, the adjective "mutsuz (unhappy)" with negative polarity according to the $\operatorname{Tr} \mathrm{L}$ lexicon should be treated as positive in the sentence "Ahmet mutsuz bir çocuk değil. (Ahmet is not an unhappy child.)" since it is followed by the negative "değil (it is not)". One of our polarity alteration rules reverses the polarity of all words that immediately precede the negative "değil" in a sentence ${ }^{4}$.

\section{Sentence Valence}

Our goal is to alter the sentiment of a Turkish sentence while preserving its content. This requires a means of assessing the sentiment change in the slanted versions of a sentence and beforehand computing their sentence valences. Literature has proposed different methods to calculate sentence valence using word polarities such as summing valence scores of all words in a sentence (Inkpen et al., 2004) or using the valence score of a present word with a strong valence. We first examined whether computing sentence valence by summing word polarities, a commonly used approach in English, is applicable to Turkish.

We conducted a formal experiment with 24 participants, all of which are Turkish native speakers. The participants were presented with 20 sentences and asked to classify each sentence as positive, negative, or neutral based on its content. The sentences, originally published in academic proses or newspapers, were manually selected from the Turkish National Corpus (Aksan et al., 2012). A strong attention was paid to select sentences that contain at least one word within the $\operatorname{Tr} L$ lexicon. The valences of these sentences were computed by summing the word polarities as determined by our polarity detection approach $^{5}$. Unfortunately, this straightforward approach failed to classify sentences as participants did in 13 sentences. The classifications of our approach and the participants in these cases are; positive-neutral in 6 sentences, negative-neutral in

\footnotetext{
${ }^{4}$ Evaluating the reliability of our polarity detection approach and how well the polarity assignments coincide with human judgements is in our future work.

${ }^{5}$ The word polarity is +1 and -1 for positive and negative words respectively. A sentence is considered as positive if the sentence valence score $>0$ and as negative if the sentence valence score $<0$. In each sentence, less than half of the content words are annotated with a polarity.
}

3 sentences, neutral-negative in 2 sentences, and positive-negative in the remaining 2 sentences. For example, our approach classified the sentence "Bir simulasyon modelinin amaci bir problemi çözmek ya da çözümüne katkıda bulunmaktır. (The purpose of a simulation model is to solve a problem or to contribute to its solution.)" with a valence of +1 as positive, whereas the participants classified it as neutral.

One reason for the divergence in classifications might be the fact that our approach considers all words in the Turkish lexicon as having the same strength and of the first sense although senses are not given in the lexicon. Since this study revealed that sentence valences determined in this fashion do not correspond with valences as assigned by humans, we argue that slanting of texts cannot be assessed by considering only sentence valences.

\section{Generating Valence Shifted Sentences}

To explore how Turkish speakers alter the sentiment characteristics of sentences, we conducted an experiment with 19 participants where they were presented with 20 sentences and asked to generate slanted versions of these texts toward a more positive or more negative direction. The sentences along with their sentiments (i.e., positive or negative) were selected from a database of movie and product reviews. The analysis of this experiment provided a number of insights into Turkish valence shifting, the three main of which are: i) slanted versions of texts are produced via three kinds of sentential changes (lexical substitution, paraphrasing, and adverbial changes that behave as intensifiers/downtoners), ii) adverbs of certainty, probability, and quantity are often used in adverbial changes, and iii) the sentence sentiment, intended shift direction, and sentence constituents determine the kind of sentential change and where in the sentence it occurs. In this work, we limit ourselves to exploring how valence shifted Turkish sentences can be generated by lexical substitution and adverbial changes ${ }^{6}$.

Lexical substitution of semantically related words with different polarity strengths is a popular approach in English. Since the Turkish polarity lexicon does not provide polarity strengths and our polarity detection approach assigns the same polarity to all synonym words, substituting a word

\footnotetext{
${ }^{6}$ Generating slanted versions of Turkish texts by paraphrasing their content is left for future work.
} 
with its synonym of the same strength to slant a text is not applicable in our case. We rather substitute words with other words that share either the "similar to" or "also see" relation if any of the 6 lexical substitution rules that we constructed is applicable. Below are two representative rules:

- If the intended shift is to increase the sentence valence, then substitute a word having a reverse polarity with the sentence sentiment with a word that has the same polarity with the sentence.

- If the intended shift is to decrease the sentence valence, then substitute a word having the same polarity with the sentence sentiment with a word of the same polarity once the polarity strength of the English correspondent of the substituted word is lower than that of the replaced word according to MPQA_L.

To capture adverbial changes, we constructed 10 rules whose applicability depends on sentence constituents. We classified all certainty, probability, and quantity adverbs as intensifiers or downtoners. These adverbs are either inserted, deleted, or substituted once an adverbial rule is applied to a sentence. In the current setting, the selection of which adverb will be used among all other possibilities is determined by a language model and the adverbial rules that apply to adjectives have a precedence over those that apply to verbs. Two representative rules are shown below:

- If the sentence contains only one adjective which has the same polarity with the sentence sentiment and the intended shift is to increase the sentence valence, then insert an intensifier in front of the adjective.

- If the denominative verb of the sentence is derived from an adjective which has the same polarity with the sentence sentiment and the intended shift is to increase the sentence valence, then insert an intensifier in front of the verb.

Our approach follows a straightforward strategy for shifting sentence valences. Once a sentence and the shift direction are given, the lexical substitution rules are applied in an order until a slanted version of the sentence is produced in the intended direction. If these rules do not succeed in slanting the sentence, then the adverbial rules are applied to the sentence.
To evaluate the effectiveness of our valence shifting approach, we conducted an experiment with 15 Turkish native speakers. The participants were presented with 21 sentence pairs, where one sentence is an original product review and the other sentence is its slanted version as produced by our valence shifting approach. In total, 9 adverbial and 3 lexical substitution rules were used for generating valence shifted sentences. We asked participants to specify which sentence in a pair has a higher valence according to the given sentence sentiment. Our results demonstrated that all participants agreed that our approach achieved the intended shift in 3 sentence pairs and the majority of them agreed on that in 8 of the remaining 18 sentence pairs. This evaluation study also revealed that the adverbial rules have a higher accuracy in shifting the sentence valence as compared to that of lexical substitution rules. Among the tested adverbial rules, the one, which modifies the adjective of the sentence subject if the polarity of the adjective contrasts with the sentence sentiment, did not achieve the intended valence shift. Moreover, the performance of the lexical substitution rules was observed to be higher in cases where the "similar to" relation is utilized than the cases where the "also see" relation is used. Since this initial study left many questions unexplored regarding the applicability and accuracy of all rules that we constructed, a more comprehensive study is necessary to better predict their performances.

\section{Conclusion}

This paper has presented our initial explorations on Turkish sentence valence and our methodology for generating valence shifted sentences in accordance with these explorations. To our knowledge, our work is the first to address the problem of valence shifting in Turkish by considering word polarities. We have presented our approach for producing slanted versions of sentences by substituting words with the use of WordNet relations and taking advantage of Turkish intensifiers and downtoners. We constructed a set of rules for specifying how and when words can be substituted or intensifiers/downtoners can be used to shift the valence of a sentence in the intended direction. In the future, we will address the task of learning polarity strengths of Turkish words and the learning of paraphrase patterns from a big collection of texts to improve the performance of our approach. 


\section{References}

Yesim Aksan, Mustafa Aksan, Ahmet Koltuksuz, Taner Sezer, Umit Mersinli, Umut Ufuk, Hakan Yilmazer, Ozlem Kurtoglu, Gulsum Atasoy, Seda Oz, and Ipek Yildiz. 2012. Construction of the turkish national corpus (tnc). In Proceedings of the Language Resources and Evaluation Conference (LREC), pages 3223-3227.

Andrea Esuli and Fabrizio Sebastiani. 2006. Sentiwordnet: A publicly available lexical resource for opinion mining. In Proceedings of the Language Resources and Evaluation Conference (LREC), pages 417-422.

Mary Gardiner and Mak Dras. 2012. Valence shifting: Is it a valid task? In Proceedings of the Australian Language Technology Association Workshop, pages 42-51.

Marco Guerini, Carlo Strapparava, and Oliviero Stock. 2008. Valentino: A tool for valence shifting of natural language text. In Proceedings of the Language Resources and Evaluation Conference (LREC), pages 243-246.

Diana Zaiu Inkpen, Olga Feiguina, and Graeme Hirst. 2004. Generating more-positive or more-negative text. In Proceedings of the AAAI Spring Symposium on Exploring Attitude and Affect in Text: Theories and Applications.

MPQA. 2014. Mpqa opinion corpus, http://mpqa.cs.pitt.edu/.

Cüneyd Murad Özsert and Arzucan Özgür. 2013. Word polarity detection using a multilingual approach. In Proceedings of the CicLing Conference, pages $75-82$.

Philip Stone, Dexter Dunphy, Marshall Smith, and Daniel Ogilvie. 1966. General Inquirer: A Computer Approach to Content Analysis. MIT Press.

Simon Whitehead and Lawrence Cavedon. 2010. Generating shifting sentiment for a conversational agent. In Proceedings of the NAACL HLT 2010 Workshop on Computational Approaches to Analysis and Generation of Emotion in Text (CAAGET), pages 89-97. 